\title{
A Conversation Between Ignasi Carrió and Ken Herrmann
}

\author{
Ignasi Carrió ${ }^{1}$ and Ken Herrmann ${ }^{2}$
}

${ }^{1}$ Hospital de la Santa Creu i Sant Pau, Barcelona, Spain; and ${ }^{2}$ Universitätsklinikum Essen, Essen, Germany

$\mathbf{K}$ the Universitätsklinikum Essen (Germany), talked with Ignasi Carrió, MD, a professor of Radiology and Nuclear Medicine at the Autonomous University of Barcelona (Spain) and director of the Department of Nuclear Medicine at Hospital Sant Pau in Barcelona. Dr. Carrió is a former president of the European Association of Nuclear Medicine (EANM) and immediate-past-editor-in-chief of the European Journal of Nuclear Medicine and Molecular Imaging (EJNMMI). He is the author of more than 250 peerreviewed articles and editor or coeditor of 6 books. His research interests include nuclear cardiology, PET/CT and SPECT/CT across a range of cancer diagnoses and treatments, radiotracers in neurology and psychiatry, and small-animal imaging. He is a frequent lecturer and participant in international scientific panels and symposia.

Dr. Herrmann: Thank you very much for taking the time. Can you give us a little professional background information?

Dr. Carrió: I graduated from medical school in Barcelona in 1973. I was first trained in oncology and became interested in targeted therapy with radionuclides. At that time, when nuclear medicine did not exist as a specialty in Spain, I discovered the great potential of radionuclides and labeled molecules for understanding physiology and disease states. In the early 1980s, I traveled to Boston, where William Strauss was the director of the Department of Nuclear Medicine at the Massachusetts General Hospital. Bill was an inspiring mentor. This was early during the time when cardiac SPECT imaging was being clinically introduced. The use of antimyosin monoclonal antibodies to detect myocardial necrosis was also very interesting, and I started my research using such antibodies to assess myocardial damage in cardiomyopathies and heart transplantation. Back in Barcelona I expanded my cardiovascular research, including cardiac function, cardiotoxicity, and other areas of cardiovascular research, including atherosclerosis.

I founded the nuclear medicine department at the Hospital Sant Pau in 1987, where I spent my entire career. At the same time, nuclear medicine was recognized as an independent medical specialty in Spain. Over the years, with the clinical implementation of PET and PET/CT and the clinical impact of PET/CT in oncology, my focus went back to oncology-the key area of interest for current residents.

I also became involved in the EANM and served as president between 2002 and 2004. From 2004 to 2018, I served as editor-inchief of EJNMMI, where I started and developed the EJNMMI family of journals.

Dr. Herrmann: I did not know that you originally came from oncology, and it's interesting to see you circling back. Based on

COPYRIGHT @ 2020 by the Society of Nuclear Medicine and Molecular Imaging. your strong background in cardiology, how do you see the future of nuclear cardiology?

Dr. Carrió: Cardiac SPECT imaging is now declining in Europe. This is because of the use of CT angiography and MR perfusion studies that are often performed by the cardiologists and, therefore, may become the preferred test. PET can offer a quantitative assessment of myocardial blood flow and flow reserve, but its use is limited by the lack of fluorine-labeled tracers.

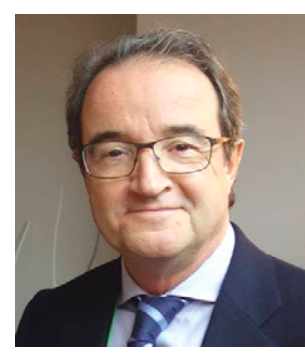

Ignasi Carrió, MD Nuclear medicine has contributed to the understanding of natural disease history in this area, but clinically relevant imaging tests to identify patients at risk for cardiac events are still lacking. Plaque imaging is limited by small targets and faint signals. We need closer partnerships with referring physicians. It is important to remain focused on the clinically relevant issues. Relevant tests must become part of clinical guidelines. It requires political skill to establish standardization and have recognition at the global level.

Dr. Herrmann: Cadmium-zinc-telluride cameras are coming, and an ${ }^{18} \mathrm{~F}$-labeled perfusion tracer is in a phase 3 clinical trial. Do you think we will recover lost ground? Or do you think it's too late?

Dr. Carrió: It is not too late. But as we see in other areas of medicine, we need to understand how medicine is evolving. In cardiovascular medicine, we must create evidence for cost-effectiveness within a reasonable timeframe. I am really looking forward to having a fluorine-labeled perfusion tracer.

Dr. Herrmann: Do you think artificial intelligence (AI) can help nuclear medicine?

Dr. Carrió: We must learn to use AI to help us in the diagnostic process-not just sitting in a reading room and waiting for AI to take over image interpretation. In order to learn, we must incorporate AI tools into our interpretation.

Dr. Herrmann: Nuclear cardiology tests are already quite standardized in terms of analysis. That's why I wonder if this might make it easier for AI to gain traction and really help us.

Dr. Carrió: Yes. Recent papers show that AI tools can perform as well as clinical reading. And that's true. But that entails a risk for us. The risk is that AI can take over an important part of our entire professional work.

Dr. Herrmann: And what about oncology?

Dr. Carrió: We must understand how oncology is evolving. Tumors are characterized genomically and molecularly from resected specimens or via liquid biopsy. This potentially enables systemic-targeted therapies. But local treatment is the first option. When staging defines therapy, imaging will remain key for assessment of response to treatment or to assess a recurrence and guide systemic 
therapy. Liquid biopsy has a huge potential and may reduce the need for imaging. The question is, what should we do in the future to stay relevant? We must demonstrate added value. Based on genomic data one can have low- and high-risk patients. Depending on risk stratification, imaging may be helpful to further stratify and tailor therapy.

Dr. Herrmann: So, one of the key phrases would be risk stratification?

Dr. Carrió: I think so. Then we must be present at tumor boards and be part of the decision-making process beyond image interpretation. This is about acting and being recognized as clinicians.

Dr. Herrmann: I agree completely. We talked about trends in imaging. One of our challenges in nuclear medicine is our lack of speed; we are often a bit slow. How can we accelerate the translation of new applications and also include them in our training curricula?

Dr. Carrió: The major hurdle in translating new tracers into the clinic has historically been an excessive regulatory framework designed for regular drugs. So how can we overcome this? On one hand it is important to partner with powerful medical specialties that could help us in such a process. At the level of the European Commission, oncology has the muscle, track record, and social impact to move the process forward. We also need to raise industry interest and support. Without appropriate and realistic business models it is unlikely that new tracers can make it into the clinic. New tracers must be vetted for relevance by clinicians and for financial viability by health economists and in-
When a new tracer is offered or proposed to be sold at $€ 1000$, it simply will not be used. That has happened with some tracers. If the market size is predictable in terms of incidence and prevalence of a given cancer, a reasonable pricing should be achievable.

Dr. Herrmann: An important topic is training. You started off in oncology and ended up in nuclear medicine. Now, the new generation of nuclear medicine practitioners is really trained only in nuclear medicine. You mentioned oncology, the future. You need to know more. We need to reach out. What do you think? How should this be reflected in the training?

Dr. Carrió: I strongly believe that nuclear medicine is a medical specialty, not an imaging specialty. It has its own body of knowledge and its own clinical and research skill requirements. It thus must remain an independent specialty, and new specialists should be trained accordingly. Our residency program consists of 4 years with the necessary common training. There is, of course, content in common with other fields, such as radiology, but the 4-y curriculum is independent. Nuclear medicine is established in most hospitals as an independent department. The new generation of specialists is hired as medical staff in these departments. I am not against cross-training oncologists, for example, as long as we keep nuclear medicine as an independent specialty. Or if we wish to train cardiologists who are now specializing in cardiac imaging to make proper use of myocardial SPECT, I'm not against that.

Dr. Herrmann: Why don't we implement an oncology fellowship for nuclear medicine fellows who have experience in thera-

"I strongly believe that nuclear medicine is a medical specialty, not an imaging specialty."

dustry. We have a tendency to base our reasoning and expectations on wishful thinking - and then reality sometimes comes a little bit too late.

Dr. Herrmann: I have 2 follow-up questions. Novartis and a couple of other big pharmaceutical companies have entered the nuclear medicine market. Do you think that this is going to be a great chance and opportunity or a threat?

Dr. Carrió: They have the power and the skills to do that. What sometimes worries me is that revenue from a single product may develop slowly. Thus, they may not invest in a subsequent product. But that's life in the business. The good news is that we now have powerful companies to work with.

Dr. Herrmann: There is a clear business case for therapy, but developing a new imaging compound costs around \$20 million for Food and Drug Administration approval and possible reimbursement. A single dose of ${ }^{18} \mathrm{~F}-F D G$ costs between $\$ 110$ and $\$ 120$. Thus, revenues are modest. Do you think there will ever be successful business cases for imaging alone, or do you think that imaging can be financially successful only if it's linked to a therapy?

Dr. Carrió: For any radiopharmaceutical to be applied to a large patient population and therefore to create a successful business, full registration for human use is a necessity. European and U.S. legislation is unlikely to change in this regard.

Dr. Herrmann: So, what needs to change to arrive at a sustainable business model? What is your opinion on development costs and reimbursement of new tracers?

Dr. Carrió: FDG has set the stage, and most health-care systems understand that this is the standard for PET and PET/CT. nostics? They could undergo 6-12 months of training to make sure that they know how to include our therapies in the appropriate overall theranostic context and are familiar with side effects and how to treat them.

Dr. Carrió: It sounds good to me, but it has one difficulty. Who hires someone who is trained in theranostics and on which basis? Usually, people are hired because they are nuclear medicine specialists.

Dr. Herrmann: Of course, they have to do nuclear medicine first.

Dr. Carrió: Oh, yes. That would be very nice. I would hire such a person

Dr. Herrmann: I would, too! When you look at the last 2 years, dynamic innovation in nuclear medicine has come from Europe. However, the revenues are in the United States. How can we as Europeans make sure that the future of our field will be also in the future driven from Europe?

Dr. Carrió: Europe is important, but science knows no borders. Discovery and innovation can show up anywhere around the world. It is true that what becomes clinical practice in Europe has a global influence and, in the long run, may determine clinical practice at the global level. On the other hand, it is important again to recognize the way in which nuclear medicine is established in Europe. The success comes from independent clinical departments that favor relevant research and clinical translation of our diagnostic and therapeutic concepts.

Dr. Herrmann: You served as editor-in-chief of EJNMMI for 14 years. You took over the journal with an impact factor of roughly 
3.3, and, just before your retirement as editor-in-chief, you achieved an impact factor of 7.7. What were the key drivers in developing this journal from a medium range to one of the toprange journals in our field?

Dr. Carrió: First, the immediate implementation of the online system was an important factor. In retrospect, it is hard to believe that the previous editors could cope with the workload without the help of an online system. Having a well-organized and efficient editorial office has been key. During my term, I always tried to offer rapid decisions with a fast and balanced review process. Authors were highly appreciative that the journal dealt with their papers expeditiously. Finally, it is important for any editor to attract key papers, good papers. And a little bit of good luck is always welcome.

Dr. Herrmann: Success is based on hard work. How much work was it?

Dr. Carrió: Well, you know, I would like to say that I strongly believe that editors of scientific journals must be professionally appointed. It's an everyday job without holidays. Articles are produced everywhere and around the clock. In my case, it required 2 $\mathrm{h}$ per day, every single day in the calendar year.

Dr. Herrmann: The strategy of The Journal of Nuclear Medicine, with a single dedicated journal, is different from that of EJNMMI. You mentioned the journal family that you created. Do you believe that creating the family of journals was the right decision?

Dr. Carrió: Our field requires and deserves many journals. We are fortunate to have an extremely bright and productive scientific community. It is important to note that, in comparison with other areas of medicine with much larger numbers of specialists, nuclear medicine produces a significantly larger number of manuscripts, many with higher scientific impact. In our case, to deal with the ever-increasing number of submissions, we decided to start with a family of journals to accommodate scientific production in the different areas within or related to nuclear medicine. I believe it has proven to be a successful approach.

I do not know the exact mechanism for such a success story, but take, for example, basic science and physics articles. In my time as editor, I tried to keep for the "mother" journal the basic science articles that were deemed to have significant clinical relevance or clinical impact. The remaining articles could eventually be published in the other more focused journals in the family, either the research or the physics journal. After a few years, the research journal now has an impact factor of around 3, with around 3.5 for the physics journal.

Dr. Herrmann: Looking back to when you finished medical school, who was the most important person during your medical career?

Dr. Carrió: Bill Strauss was an illuminating scientist and mentor, and what I learned from him has been with me for my entire career. Peter Ell has also been most influential. I learned from him that top science is compatible and has to go together with wellorganized professional societies. He was the founder and architect behind the EANM and was the founding editor-in-chief of the EJNMMI, who brought the journal to the forefront of the field. These 2 people had a big influence on me.

Dr. Herrmann: Which situation shaped you most in your professional development?

Dr. Carrió: That's a difficult one. I can remember being in the early morning session at the nuclear medicine department at Mass General while the room was dark (we were still reading transparent films on light boxes). Suddenly I felt the emotion of being able to think freely about pathophysiology and speak with a broad view to other specialists. That day I decided that this was going to be my field. I still can remember the feeling. As another example, on the first day as EJNMMI editor when I received the first article on my website, I immediately felt the responsibility and magnitude of the work I was going to face.

Dr. Herrmann: Looking back, is there anything you would do differently?

Dr. Carrió: I would not waste my time trying to convince the already converted. In other words, I would go less to nuclear medicine meetings and more to meetings by other specialtieseasy to say but difficult to do.

Dr. Herrmann: What is your most important advice to young nuclear medicine physicians?

Dr. Carrió: I always tell them to think independently and not take current concepts for granted-to challenge what is already established. And I tell them that nothing is impossible. Then I tell them that they must keep their passion. If you remain in an office just reading images, you are dead.

Dr. Herrmann: Thank you very much for dedicating your time to our readers. 